\title{
Efficacy and Cost-Efficacy of Biologic Therapies for Moderate to Severe Psoriasis: A Meta-Analysis and Cost-Efficacy Analysis Using the Intention-to-Treat Principle
}

\author{
Ching-Chi Chi ${ }^{1,2}$ and Shu-Hui Wang ${ }^{3,4}$ \\ ${ }^{1}$ Department of Dermatology and Centre for Evidence-Based Medicine, Chang Gung Memorial Hospital, Chiayi 61363, Taiwan \\ ${ }^{2}$ College of Medicine, Chang Gung University, Taoyuan 33302, Taiwan \\ ${ }^{3}$ Department of Dermatology, Far Eastern Memorial Hospital, Banciao, New Taipei 22060, Taiwan \\ ${ }^{4}$ Oriental Institute of Technology, New Taipei 22061, Taiwan
}

Correspondence should be addressed to Shu-Hui Wang; dermawang@hotmail.com

Received 30 April 2013; Revised 13 November 2013; Accepted 18 November 2013; Published 28 January 2014

Academic Editor: Yoshio Ishibashi

Copyright (C) 2014 C.-C. Chi and S.-H. Wang. This is an open access article distributed under the Creative Commons Attribution License, which permits unrestricted use, distribution, and reproduction in any medium, provided the original work is properly cited.

Background. Compared to conventional therapies, biologics are more effective but expensive in treating psoriasis. Objective. To evaluate the efficacy and cost-efficacy of biologic therapies for psoriasis. Methods. We conducted a meta-analysis to calculate the efficacy of etanercept, adalimumab, infliximab, and ustekinumab for at least 75\% reduction in the Psoriasis Area and Severity Index score (PASI 75) and Physician's Global Assessment clear/minimal (PGA 0/1). The cost-efficacy was assessed by calculating the incremental cost-effectiveness ratio (ICER) per subject achieving PASI 75 and PGA 0/1. Results. The incremental efficacy regarding PASI 75 was 55\% (95\% confidence interval (95\% CI) 38\%-72\%), 63\% (95\% CI 59\%-67\%), 71\% (95\% CI 67\%-76\%), 67\% (95\% CI $62 \%-73 \%)$, and $72 \%$ (95\% CI 68\%-75\%) for etanercept, adalimumab, infliximab, and ustekinumab $45 \mathrm{mg}$ and $90 \mathrm{mg}$, respectively. The corresponding 6-month ICER regarding PASI 75 was $\$ 32,643$ (best case $\$ 24,936$; worst case $\$ 47,246$ ), $\$ 21,315$ (best case $\$ 20,043$; worst case $\$ 22,760$ ), $\$ 27,782$ (best case $\$ 25,954$; worst case $\$ 29,440$ ), $\$ 25,055$ (best case $\$ 22,996$; worst case $\$ 27,075$ ), and $\$ 46,630$ (best case $\$ 44,765$; worst case $\$ 49,373$ ), respectively. The results regarding PGA 0/1 were similar. Conclusions. Infliximab and ustekinumab $90 \mathrm{mg}$ had the highest efficacy. Meanwhile, adalimumab had the best cost-efficacy, followed by ustekinumab $45 \mathrm{mg}$ and infliximab.

\section{Background}

Psoriasis is a chronic inflammatory disease affecting $1-3 \%$ of the general population and incurs a considerable economic burden [1]. A number of biologics have been introduced for treating moderate to severe psoriasis. Etanercept is a fusion protein that binds to and neutralizes tumor necrosis factor (TNF) [2]. Adalimumab is a recombinant monoclonal antibody that binds to TNF and blocks its interaction from TNF receptors [3]. Infliximab is a chimeric monoclonal antibody which binds and neutralizes TNF [4]. Ustekinumab is a monoclonal antibody against the p40 subunit of the IL-12 and IL-23 cytokines which are involved in inflammatory and immune responses [5]. Short-term trials on these biologics showed that $47 \%-88 \%$ of the participants achieved at least $75 \%$ reduction in the Psoriasis Area and Severity Index score (PASI 75) after treatment for 10 to 16 weeks [2-5].

Biologics therapies for psoriasis are expensive. Based on the US drug price in April 2010 [6], the 6 month drug costs are $\$ 17,954, \$ 13,429, \$ 19,725, \$ 16,787$, and $\$ 33,574$ for the etanercept, adalimumab, infliximab (for a person weighing $81-100 \mathrm{~kg}$ ), ustekinumab $45 \mathrm{mg}$, and ustekinumab $90 \mathrm{mg}$ regimens approved by the Food and Drug Administration (FDA) (see below), respectively. Healthcare payers therefore often have an eligibility criterion for the reimbursement of biologics therapies. Patients with moderate to severe psoriasis (defined as involvement of greater than $5 \%$ body surface area 
or involvement of $\leq 5 \%$ body surface area affecting sensitive areas or areas that significantly impact daily function (e.g., palms, soles, head, neck, or genitalia) are eligible for reimbursement if the psoriasis has not responded to phototherapy and systemic agents (such as acitretin, methotrexate, and cyclosporine) or if the patients are intolerant of, or have a contraindication to, these treatments [7].

The drug costs for treating psoriasis in the US have increased by $30 \%$ from 2000 to 2008 , with a major contribution from biologics [1]. The increasing drug spending leads to an economic burden of healthcare systems. The objective of this study was to use the best evidence to assess the efficacy and cost-efficacy of biologic therapies for treating moderate to severe psoriasis. It is our hope that this will assist in efficient allocation of limited resources in treating psoriasis. We did not analyze conventional therapies in this study as biologic therapies are primarily used as second-line treatments when conventional therapies fail or are contraindicated.

\section{Methods}

2.1. Meta-Analysis. We performed a meta-analysis of randomized controlled trials using the intention-to-treat (ITT) principle to assess the efficacy of etanercept, adalimumab, infliximab, and ustekinumab in treating psoriasis. We searched the Cochrane Central Register of Controlled Trials, MEDLINE, and EMBASE for relevant studies on November 23, 2012. The inclusion criteria of studies were randomized placebo-controlled trials which assessed the efficacy of etanercept, adalimumab, infliximab, and ustekinumab in treating moderate to severe psoriasis in adults by using the FDAapproved regimens for at least 6 months.

We included trials that adhered to the regimens approved by the US FDA. Trials that did not use an approved regimen were excluded. If a multiarm placebo-controlled trial contained an arm using an approved regimen and another using an unapproved regimen, we extracted relevant data from the arm using the approved regimen and the placebo arm. The approved etanercept regimen for treating psoriasis is $50 \mathrm{mg}$ twice weekly in the first 12 weeks, followed by $50 \mathrm{mg}$ once weekly or $25 \mathrm{mg}$ twice weekly [2]. The approved adalimumab regimen is $80 \mathrm{mg}$ at week 0 , followed by $40 \mathrm{mg}$ every other week [3]. The approved infliximab regimen is $5 \mathrm{mg} / \mathrm{kg}$ administered at weeks 0,2 , and 6 , followed by $5 \mathrm{mg} / \mathrm{kg}$ every 8 weeks thereafter [4]. The approved ustekinumab regimen is $45 \mathrm{mg}$ (or $90 \mathrm{mg}$ for patients weighing over $100 \mathrm{~kg}$ ) at week 0 and week 4, followed by $45 \mathrm{mg}$ (or $90 \mathrm{mg}$ for patients weighing over $100 \mathrm{~kg}$ ) every 12 weeks [5].

The primary efficacy outcome was the proportion of participants achieving PASI 75 at month 6 (week 24-28 were acceptable). We built a decision tree for analysis as shown in Figure 1. The proportion of participants achieving PASI 75 was $P_{b}$ and $P_{c}$ in the biologics and placebo groups, respectively. The secondary efficacy outcome was the proportion of participants achieving Physician's Global Assessment clear or minimal (PGA 0/1) at month 6. We calculated the outcomes based on all randomized participants, that is, ITT analysis. All randomized participants with missing

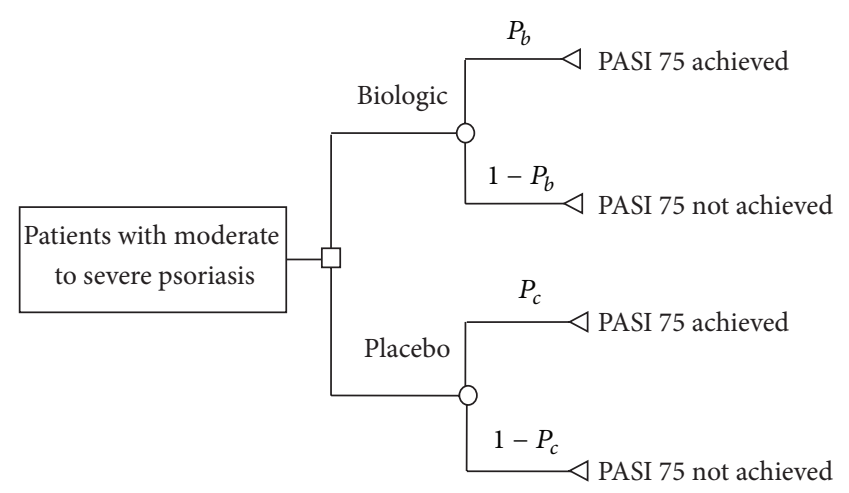

FIGURE 1: Decision tree.

outcome data were considered treatment failure. If a trial did not have data on PASI 75 and PGA 0/1 response after 6 months' use of placebo because the placebo groups were switched to biologics treatment before month 6, we used the last observation carried forward approach to estimate the outcomes. For example, if the placebo group was switched to biologic treatments at week 12, we used the PASI 75 and PGA 0/1 data at week 12 as the estimated efficacy after 6 months' use of placebo.

We defined incremental efficacy as the absolute increase in the proportion of participants achieving a prespecified outcome after a biologic therapy when compared to placebo, that is, $P_{b}-P_{c}$ (see Figure 1). We calculated the 6-month incremental efficacy of each biologic regimen for PASI 75 and PGA 0/1 response, respectively. When more than one trial were available for an outcome, we applied a meta-analysis technique to calculate the pooled efficacy and 95\% confidence interval (CI) by using the DerSimonian and Laird randomeffects model [8]. The Review Manager 5.1 (Nordic Cochrane Centre, Cochrane Collaboration, Copenhagen, Denmark, 2011) was used for meta-analysis.

2.2. Cost-Efficacy Analysis. For cost-efficacy analysis, we considered the direct drug costs of the approved regimen based on the US drug price in April 2010 [6]. The direct costs are Cost $_{b}$ and Cost $c$ in the biologics and placebo groups, respectively. The costs of placebo were assumed to be nil. For infliximab, we assumed a patient bodyweight of $81-100 \mathrm{~kg}$ and wasting of remaining vial after use (one vial contains $100 \mathrm{mg}$ infliximab). We assessed the cost-efficacy by calculating the incremental cost-effectiveness ratio (ICER), which was the ratio of the increase in costs to the efficacy, that is, $\left(\right.$ Cost $_{b}-$ Cost $\left._{c}\right) /\left(P_{b}-P_{c}\right)$. In other words, the ICER was the average cost for one participant to achieve a prespecified outcome. The lower the ICER was, the more cost-effective a biologic therapy was.

We calculated the 6-month (24 weeks) base case ICERs of each biologic therapy according to the incremental efficacy when compared to placebo in terms of PASI 75 and PGA $0 / 1$. We also calculated the worst and best case ICERs based on the lower and upper 95\% confidence limits of the incremental efficacy, respectively. The range between the worst and best case ICERs can be regarded as the 95\% CI 


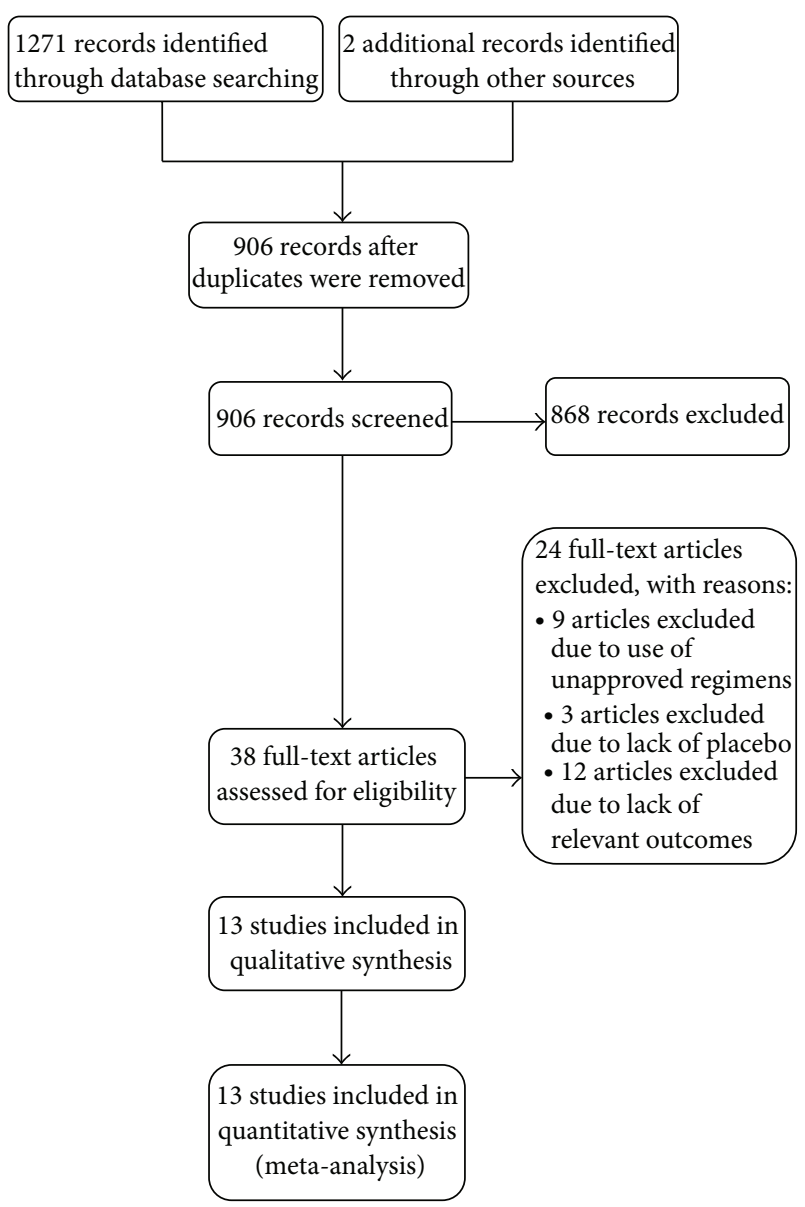

Figure 2: Study flow diagram.

of the ICER. In addition, we used the base case data to conduct an incremental analysis after excluding the least cost-effective biologic therapy and calculated the incremental costs per additional PASI 75 or PGA 0/1 responder between the remaining biologic therapies.

\section{Results}

3.1. Meta-Analysis. As shown in Figure 2, 1271 records were identified through searching the databases and 2 additional records were obtained from a pharmaceutical company. After removal of duplicates and exclusion due to use of unapproved regimens, lack of placebo, or relevant outcomes, 13 trials with a total of 5309 participants were included [9-21]. All the included trials were of high quality when appraised by using the Cochrane Collaboration's tool for assessing risk of bias in randomized trials [8]. The efficacy outcomes of the included trials are summarized in Table 1 . The meta-analysis (Figure 3) found that the pooled incremental efficacy of PASI 75 response was 55\% (95\% CI 38\%-72\%), 63\% (95\% CI 59\%$67 \%$ ), 71\% (95\% CI 67\%-76\%), 67\% (95\% CI 62\%-73\%), and $72 \%$ (95\% CI $68 \%-75 \%)$ for etanercept, adalimumab, infliximab, ustekinumab $45 \mathrm{mg}$, and ustekinumab $90 \mathrm{mg}$, respectively. The pooled incremental efficacy of PGA 0/1 response was 58\% (95\% CI 45\%-71\%), 56\% (95\% CI 52\%$59 \%), 69 \%$ (95\% CI 63\%-76\%), 58\% (95\% CI 51\%-64\%), and $62 \%$ (95\% CI 58\%-66\%) for etanercept, adalimumab, infliximab, ustekinumab $45 \mathrm{mg}$, and ustekinumab $90 \mathrm{mg}$, respectively (Figure 4).

3.2. Cost-Efficacy. Based on the ICER as to PASI 75 response (Table 2), adalimumab had the best cost-efficacy $(\$ 21,315$ in the base case, $\$ 20,043$ in the best case, and $\$ 22,760$ in the worst case), followed by ustekinumab $45 \mathrm{mg}$ ( $\$ 25,055$ in the base case, $\$ 22,996$ in the best case, and $\$ 27,075$ in the worst case) and infliximab ( $\$ 27,782$ in the base case, $\$ 25,954$ in the best case, and $\$ 29,440$ in the worst case). For etanercept, the 6 -mo ICER was $\$ 32,643$ in the base case, $\$ 24,936$ in the best case, and $\$ 47,246$ in the worst case. Ustekinumab $90 \mathrm{mg}$ had the highest 6-mo ICER ( $\$ 46,630$ in the base case, $\$ 44,765$ in the best case, and $\$ 49,373$ in the worst case).

Based on PGA 0/1 response (Table 3), adalimumab had the most favorable 6-month ICER (adalimumab: $\$ 23,980$ in the base case, $\$ 22,760$ in the best case, and $\$ 25,824$ in the worst case), followed by infliximab $(\$ 28,587$ in the base case, $\$ 25,954$ in the best case, and $\$ 31,310$ in the worst case) and ustekinumab $45 \mathrm{mg}(\$ 28,943$ in the base case, $\$ 26,229$ in the best case, and $\$ 32,915$ in the worst case). Etanercept had a wide $95 \%$ CI of a 6 -month ICER (base case $\$ 30,954$; best case $\$ 25,287$; worst case $\$ 39,897$ ) and overlapped with adalimumab and ustekinumab $45 \mathrm{mg}$. Ustekinumab $90 \mathrm{mg}$ had the highest 6-month ICER of PGA 0/1 (base case \$54,151; best case $\$ 50,869$; worst case $\$ 57,886$ ).

The base case incremental analysis (Table 4) showed when considering PASI 75 response, etanercept was dominated. Adalimumab was likely to be the most cost-effective, with a cost of $\$ 21,315$ per PASI 75 responder when compared to placebo. Ustekinumab $45 \mathrm{mg}$ had a cost of $\$ 83,950$ per additional PASI 75 responder when compared to adalimumab, while infliximab had a cost of $\$ 68,175$ per additional PASI 75 responder when compared to ustekinumab $45 \mathrm{mg}$. Ustekinumab $90 \mathrm{mg}$ had a cost of $\$ 1,384,900$ per additional PASI 75 responder when compared to infliximab. On the other hand, when considering PGA 0/1 response, adalimumab was likely to be the most cost-effective biologic therapy, with a cost of $\$ 23,980$ per PASI 75 responder when compared to placebo. Ustekinumab $45 \mathrm{mg}$ had a cost of $\$ 167,900$ per additional PGA 0/1 responder when compared with adalimumab. Compared to ustekinumab $45 \mathrm{mg}$, ustekinumab $90 \mathrm{mg}$ and infliximab had a cost of $\$ 3,358,150$ and $\$ 26,709$ per additional PGA 0/1 responder, respectively.

\section{Discussion}

Psoriasis is a chronic dermatosis which cannot be cured and imposes an impact on quality of life comparable to that experienced by patients with type 2 diabetes mellitus or chronic lung disease [22]. Clinical efficacy and costefficacy are thus important in allocating limited resources for treatments. The present study assessed the 6-month efficacy and cost-efficacy of biologic therapies by examining two outcomes (PASI 75 and PGA 0/1), and can serve as a useful 


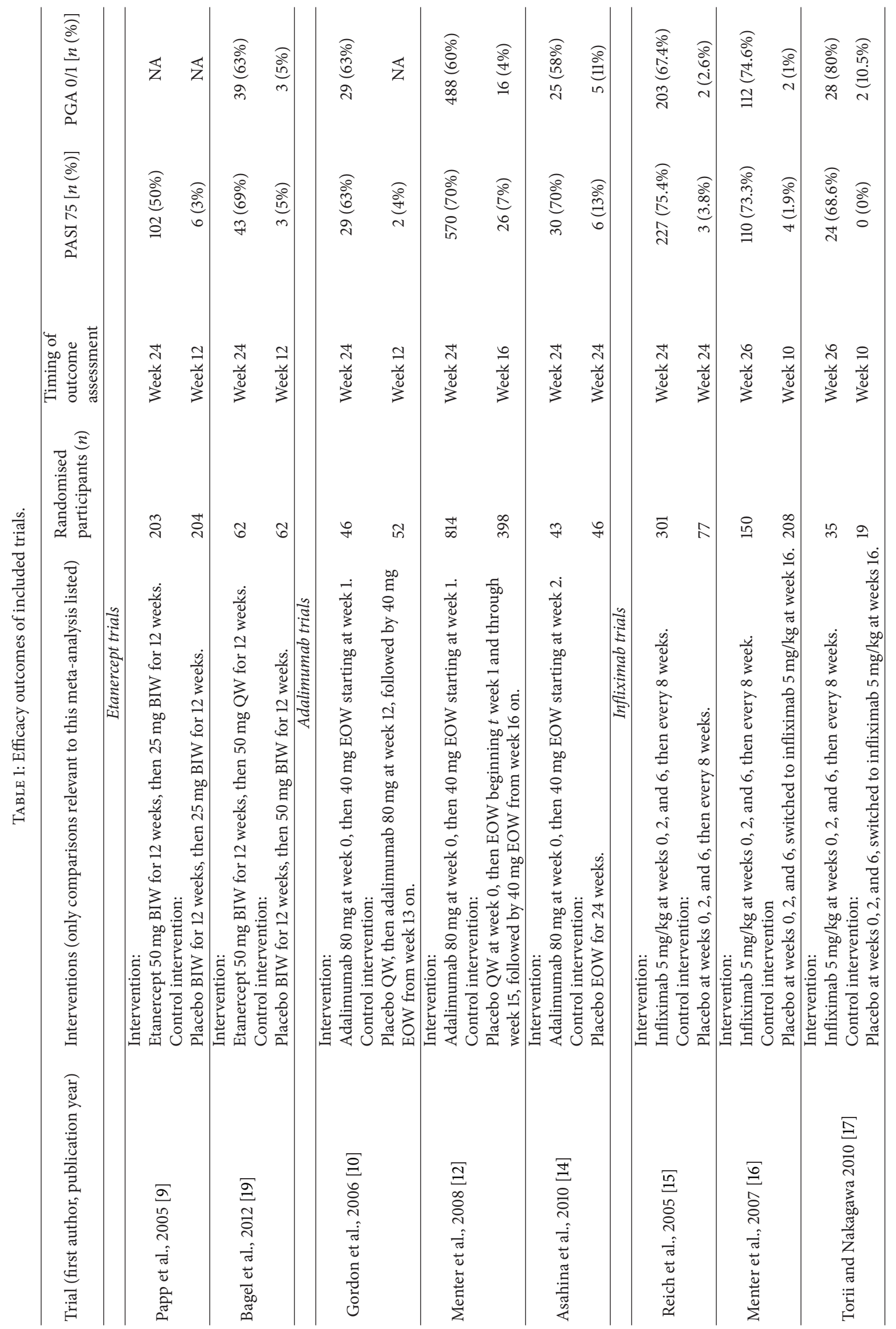




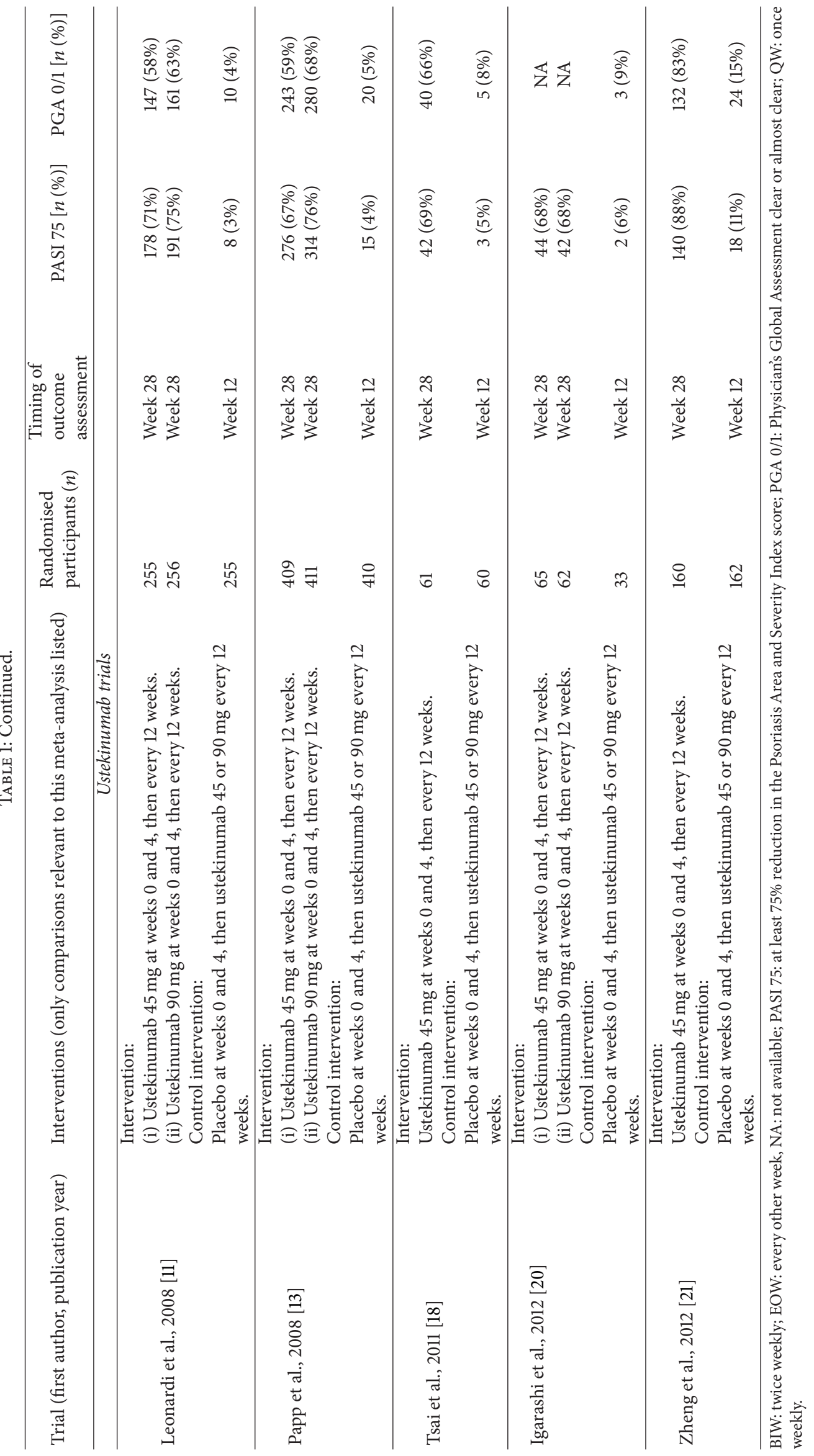




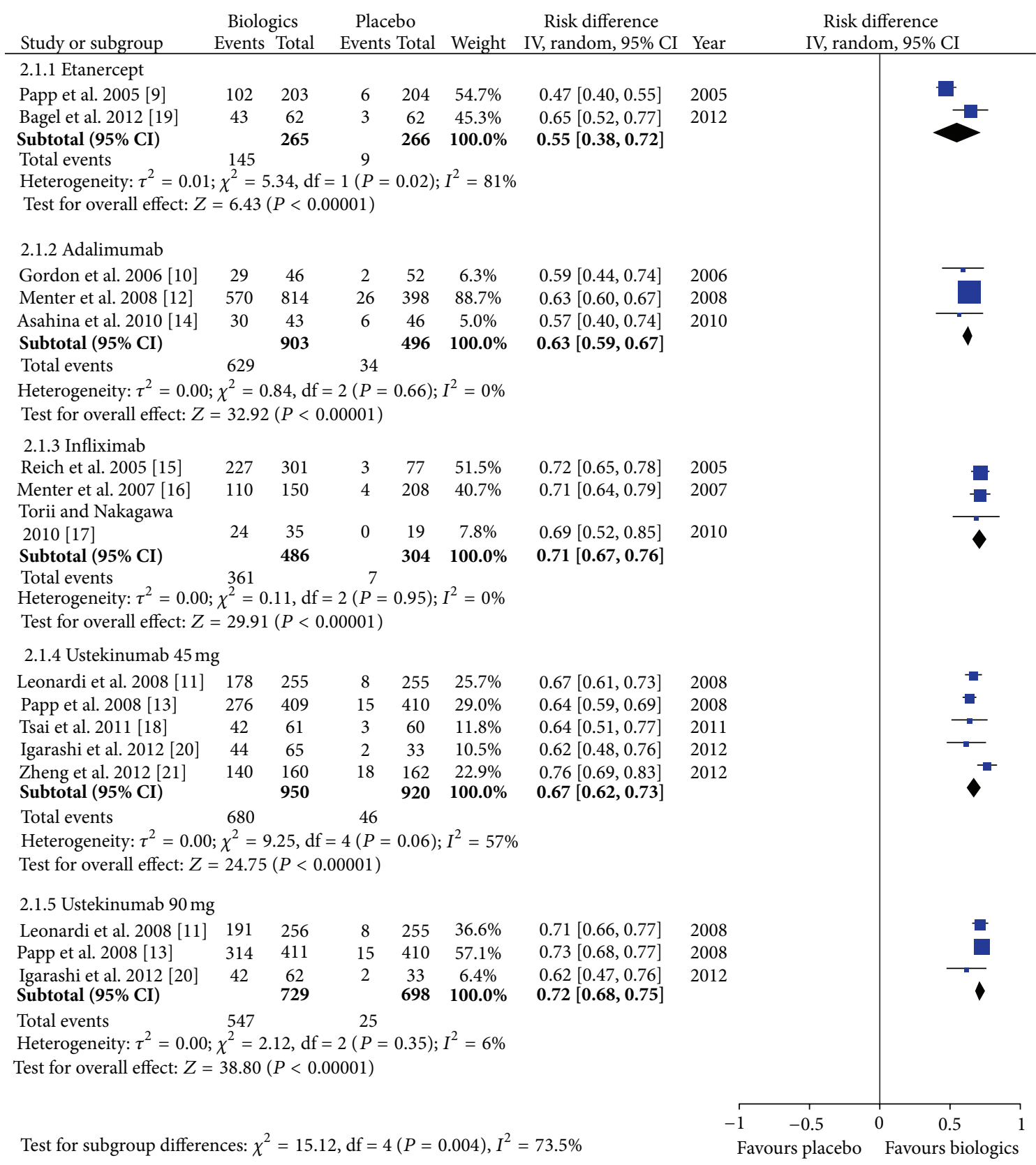

FIgUre 3: Meta-analysis based on at least 75\% reduction in the Psoriasis Area and Severity Index score.

reference for dermatologists and policy makers. Our metaanalysis revealed that infliximab and ustekinumab $90 \mathrm{mg}$ had a higher pooled incremental efficacy as to either PASI 75 or PGA 0/1 when compared to other biologics. On the other hand, adalimumab had the best cost-efficacy based on either PASI 75 or PGA 0/1, followed by ustekinumab $45 \mathrm{mg}$ and infliximab. Etanercept had a wide range of cost-efficacy estimate due to limited available data and was dominated in the incremental analysis. Ustekinumab $90 \mathrm{mg}$ had very high costs of $\$ 1,384,900$ and $\$ 3,358,150$ per additional PASI 75 and PGA 0/1 responders when compared to the next best regimen, which were above any known conventional willingness to pay threshold.
Previous economic analyses on biologics for treating psoriasis determined the efficacy based on data from shortterm endpoints at weeks 10 to 16 [23-27]. However, the efficacy may differ with time. For example, the proportion of PASI 75 responders to etanercept increased from 59\% at week 12 to $69 \%$ at week 24 [19]. The proportion of PASI 75 responders to adalimumab increased from $53 \%$ at week 12 to $64 \%$ at week 24 [10]. By using a 6-month data, our study provides a reliable reference as to intermediate-term efficacy and cost-efficacy. We originally planned to collect efficacy outcome assessed at week 52 , but could not obtain relevant data because of the fact that the length of trial was less than 52 weeks [9], discontinuation of biologic therapy for participants 


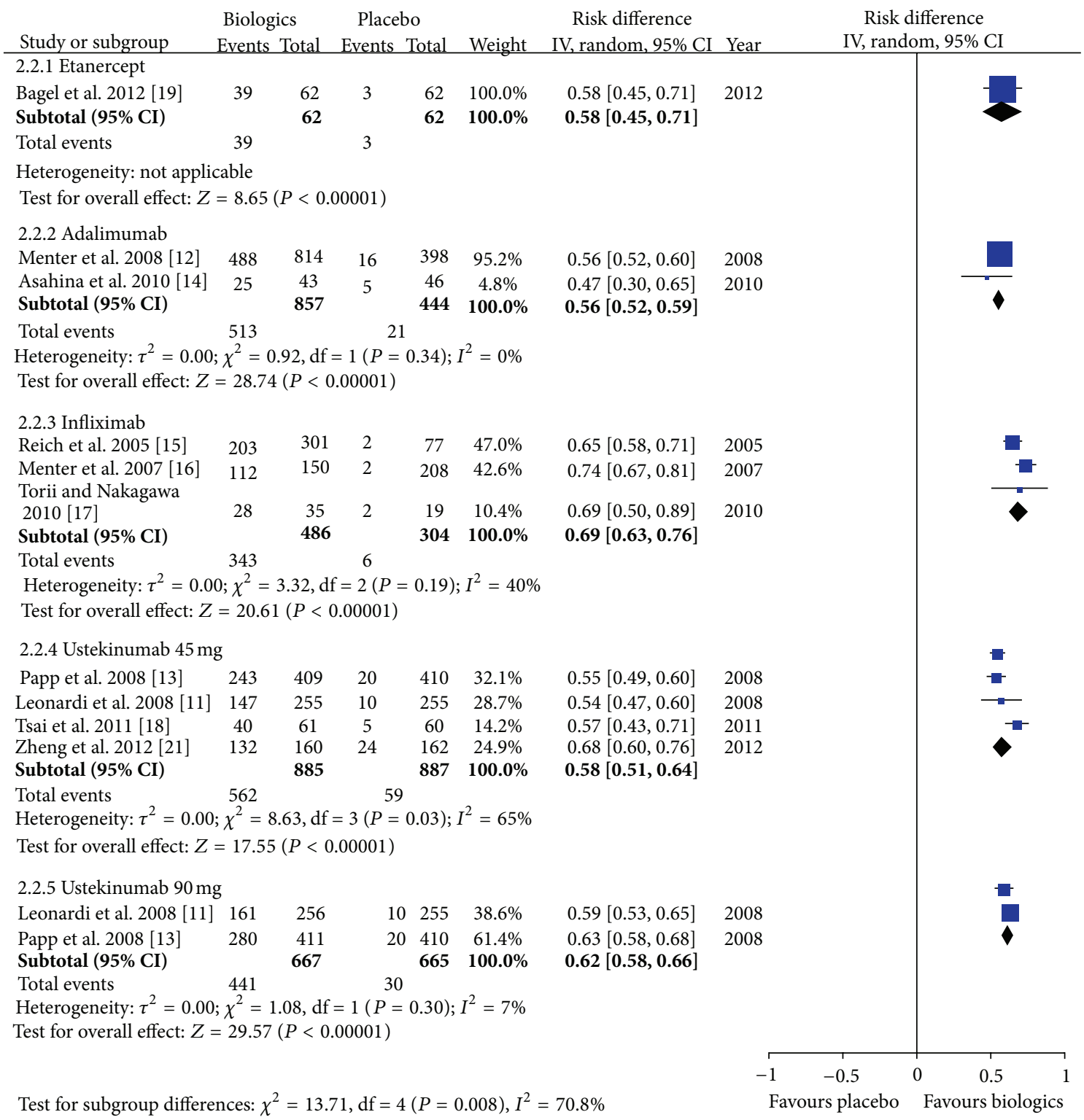

Figure 4: Meta-analysis based on Physician's Global Assessment clear or almost clear.

TABLE 2: Incremental efficacy and cost-efficacy based on at least 75\% reduction in the Psoriasis Area and Severity Index score.

\begin{tabular}{lcccccc}
\hline \multirow{2}{*}{ Biologics } & \multicolumn{3}{c}{ Pooled incremental efficacy } & \multicolumn{3}{c}{ 6-month incremental cost-effectiveness ratio } \\
& Base case & Best case & Worst case & Base case & Best case & Worst case \\
\hline Etanercept & $55 \%$ & $72 \%$ & $38 \%$ & $\$ 32,643$ & $\$ 24,936$ & $\$ 47,246$ \\
Adalimumab & $63 \%$ & $67 \%$ & $59 \%$ & $\$ 21,315$ & $\$ 20,043$ & $\$ 22,760$ \\
Infliximab & $71 \%$ & $76 \%$ & $67 \%$ & $\$ 27,782$ & $\$ 25,954$ & $\$ 29,440$ \\
Ustekinumab 45 mg & $67 \%$ & $73 \%$ & $62 \%$ & $\$ 25,055$ & $\$ 22,996$ & $\$ 27,075$ \\
Ustekinumab 90 mg & $72 \%$ & $75 \%$ & $68 \%$ & $\$ 46,630$ & $\$ 44,765$ & $\$ 49,373$ \\
\hline
\end{tabular}

with inadequate response [20], and rerandomization of participants with sustained PASI 75 response to either placebo or biologics [11-13].

Previous economic analyses only included efficacy data from trials conducted in the US and Europe where most participants were Caucasians [23-27]. The present analysis included efficacy data from four Asian trials [14, 17, 18, 20, 21], and thus it has a better generalizability in a multiethnic setting like the US.

Many trials included in this study used modified ITT analysis to assess efficacy outcomes, that is, inclusion of randomized subjects who received at least one dose of the study 
TABLE 3: Incremental efficacy and cost-efficacy based on Physician's Global Assessment clear or almost clear.

\begin{tabular}{lcccccc}
\hline \multirow{2}{*}{ Biologics } & \multicolumn{3}{c}{ Pooled incremental efficacy } & \multicolumn{3}{c}{ 6-month incremental cost-effectiveness ratio } \\
& Base case & Best case & Worst case & Base case & Best case & Worst case \\
\hline Etanercept & $58 \%$ & $71 \%$ & $45 \%$ & $\$ 30,954$ & $\$ 25,287$ & $\$ 39,897$ \\
Adalimumab & $56 \%$ & $59 \%$ & $52 \%$ & $\$ 23,980$ & $\$ 22,760$ & $\$ 25,824$ \\
Infliximab & $69 \%$ & $76 \%$ & $63 \%$ & $\$ 28,587$ & $\$ 25,954$ & $\$ 31,310$ \\
Ustekinumab 45 mg & $58 \%$ & $64 \%$ & $51 \%$ & $\$ 28,943$ & $\$ 26,229$ & $\$ 32,915$ \\
Ustekinumab 90 mg & $62 \%$ & $66 \%$ & $58 \%$ & $\$ 54,151$ & $\$ 50,869$ & $\$ 57,886$ \\
\hline
\end{tabular}

TABLE 4: Base case incremental analysis.

\begin{tabular}{lccc}
\hline & 6 -month costs & Incremental efficacy of PASI 75 response & ICER (costs per additional PASI 75 responder) \\
\hline Etanercept & $\$ 17,954$ & $55 \%$ & Dominated \\
Adalimumab & $\$ 13,429$ & $63 \%$ & $\$ 21,315^{\mathrm{a}}$ \\
Ustekinumab 45 mg & $\$ 16,787$ & $67 \%$ & $\$ 83,950^{\mathrm{b}}$ \\
Infliximab & $\$ 19,725$ & $71 \%$ & $\$ 68,175^{\mathrm{c}}$ \\
Ustekinumab 90 mg & $\$ 33,574$ & $72 \%$ & $\$ 1,384,900^{\mathrm{d}}$ \\
\hline & & Incremental efficacy of PGA 0/1 response & ICER (costs per additional PGA 0/1 responder) \\
\hline Adalimumab & $\$ 13,429$ & $56 \%$ & $\$ 23,980^{\mathrm{a}}$ \\
Ustekinumab 45 mg & $\$ 16,787$ & $58 \%$ & $\$ 167,900^{\mathrm{b}}$ \\
Etanercept & $\$ 17,954$ & $58 \%$ & Dominated \\
Ustekinumab 90 mg & $\$ 33,574$ & $62 \%$ & $\$ 3,358,150^{\mathrm{c}}$ \\
Infliximab & $\$ 19,725$ & $69 \%$ & $\$ 26,709^{\mathrm{c}}$ \\
\hline
\end{tabular}

${ }^{a}$ Compared to placebo.

${ }^{\mathrm{b}}$ Compared to adalimumab.

${ }^{\mathrm{c}}$ Compared to ustekinumab $45 \mathrm{mg}$.

${ }^{\mathrm{d}}$ Compared to infliximab.

drug in statistical analyses $[9,10]$. When assessing efficacy outcomes at month 6, some trials used perprotocol analysis, that is, only including subjects who stayed in the trials in analysis [11, 13, 15-18, 20, 21]. Both approaches excluded those lost to follow-up due to lack of efficacy from statistical analyses, which may lead to biased efficacy estimates [8]. In our meta-analysis, we recalculated all efficacy outcome data by using the ITT approach, that is, we included all randomized subjects in statistical analyses and considered those subjects with missing data as treatment failure. Therefore, our meta-analysis provides less biased efficacy estimates and best mimics actual practice where patients are able to drop out of treatment and change treatment groups.

Similar to previous economic analyses [25, 27], the present cost-efficacy analysis only considered drug costs. Other costs for administering biologics and indirect costs were not considered. The cost efficacy of infliximab will decrease if the indirect cost and the time missed from work due to intravenous administration are considered.

Biologic therapies are generally conceived to be expensive when compared to conventional therapies. However, a study revealed that introduction of biologics therapies reduced the total healthcare costs for patients who previously required long-term hospitalization for disease control, as hospitalizations were shortened or no longer needed [28]. Therefore, careful selection of patients appropriate for biologic therapies may be cost-saving on the ground of avoidance of ineffective conventional treatments, reduction of hospitalization costs, increased productivity, and reduction of indirect costs.

Although drug costs are an important concern in choosing biologics, they are not the sole determinant. Patients' unique values and circumstances should be considered in decision making [29]. For example, the total number of injections in the first 6 months' therapy is $36,13,5$, and 3 for etanercept, adalimumab, infliximab, and ustekinumab, respectively. Patients who are afraid of injections or dislike the injection pain may prefer ustekinumab therapy.

Presence of concomitant psoriatic arthritis may affect the choice of biologics. Anti-TNF $\alpha$ agents (i.e., etanercept, adalimumab, and infliximab) have established efficacy in treating psoriatic arthritis [30] and are therefore preferred in patients with concomitant psoriatic arthritis. A trial found that ustekinumab reduced symptoms and signs of psoriatic arthritis, but the administered regimen (ustekinumab $90 \mathrm{mg}$ or $63 \mathrm{mg}$ every week for 4 weeks) differed from those used in treating psoriasis [31]. Another randomized trial found that ustekinumab administered using the approved regimens improved joint pain visual analogue scale, but the efficacy appeared varying and lacked a dose-response relationship $[20]$.

\section{Conclusions}

Infliximab and ustekinumab $90 \mathrm{mg}$ had a higher pooled efficacy as to either PASI 75 or PGA $0 / 1$ when compared 
to other biologics. On the other hand, adalimumab had the lowest average costs per patient achieving PASI 75 or PGA $0 / 1$ response, followed by ustekinumab $45 \mathrm{mg}$ and infliximab. Etanercept and ustekinumab $90 \mathrm{mg}$ had an unfavorable costefficacy. Clinicians and policy-makers should consider the efficacy and cost-efficacy evidence along with patients' values and characteristics (such as presence of psoriatic arthritis) in deciding how to efficiently allocate resources in treating psoriasis.

\section{Conflict of Interests}

The authors declare that there is no conflict of interests regarding the publication of this paper.

\section{Acknowledgment}

The authors thank Professor Fenella Wojnarowska (Nuffield Department of Clinical Medicine, University of Oxford, UK) for her assistance in searching and her comments on the paper.

\section{References}

[1] V. Beyer and S. E. Wolverton, "Recent trends in systemic psoriasis treatment costs," Archives of Dermatology, vol. 146, no. 1, pp. 46-54, 2010.

[2] Prescribing InFormation for Enbrel, Immunex Corporation, Thousand Oaks, Calif, USA, 2011.

[3] Prescribing InFormation for Humira, Abbott Laboratories, North Chicago, Ill, USA, 2012.

[4] Prescribing InFormation for Remicade, Janssen Biotech, Horsham, UK, 2011.

[5] Prescribing InFormation for Stelara, Janssen Biotech, Horsham, UK, 2012.

[6] J. A. Schafer, N. K. Kjesbo, and P. P. Gleason, "Formulary review of 2 new biologic agents: tocilizumab for rheumatoid arthritis and ustekinumab for plaque psoriasis," Journal of Managed Care Pharmacy, vol. 16, no. 6, pp. 402-416, 2010.

[7] Anthem Insurance Companies, "Medical Policy on ustekinumab," October 2012, http://www.anthem.com/medicalpolicies/policies/mp_pw_c120021.htm.

[8] J. P. T. Higgins and S. Green, Cochrane Handbook for Systematic Reviews of Interventions, Wiley-Blackwell, Chichester, UK, 2008.

[9] K. A. Papp, S. Tyring, M. Lahfa et al., "A global phase III randomized controlled trial of etanercept in psoriasis: safety, efficacy, and effect of dose reduction," British Journal of Dermatology, vol. 152, no. 6, pp. 1304-1312, 2005.

[10] K. B. Gordon, R. G. Langley, C. Leonardi et al., "Clinical response to adalimumab treatment in patients with moderate to severe psoriasis: double-blind, randomized controlled trial and open-label extension study," Journal of the American Academy of Dermatology, vol. 55, no. 4, pp. 598-606, 2006.

[11] C. L. Leonardi, A. B. Kimball, K. A. Papp et al., "Efficacy and safety of ustekinumab, a human interleukin-12/23 monoclonal antibody, in patients with psoriasis: 76-week results from a randomised, double-blind, placebo-controlled trial (PHOENIX 1)," The Lancet, vol. 371, no. 9625, pp. 1665-1674, 2008.
[12] A. Menter, S. K. Tyring, K. Gordon et al., "Adalimumab therapy for moderate to severe psoriasis: a randomized, controlled phase III trial," Journal of the American Academy of Dermatology, vol. 58, no. 1, pp. 106-115, 2008.

[13] K. A. Papp, R. G. Langley, M. Lebwohl et al., "Efficacy and safety of ustekinumab, a human interleukin-12/23 monoclonal antibody, in patients with psoriasis: 52-week results from a randomised, double-blind, placebo-controlled trial (PHOENIX 2)," The Lancet, vol. 371, no. 9625, pp. 1675-1684, 2008.

[14] A. Asahina, H. Nakagawa, T. Etoh, and M. Ohtsuki, "Adalimumab in Japanese patients with moderate to severe chronic plaque psoriasis: efficacy and safety results from a Phase II/III randomized controlled study," Journal of Dermatology, vol. 37, no. 4, pp. 299-310, 2010.

[15] K. Reich, F. O. Nestle, K. Papp et al., "Infliximab induction and maintenance therapy for moderate-to-severe psoriasis: a phase III, multicentre, double-blind trial," Lancet, vol. 366, no. 9494, pp. 1367-1374, 2005.

[16] A. Menter, S. R. Feldman, G. D. Weinstein et al., "A randomized comparison of continuous vs. intermittent infliximab maintenance regimens over 1 year in the treatment of moderate-tosevere plaque psoriasis," Journal of the American Academy of Dermatology, vol. 56, no. 1, pp. 31.e1-31.e15, 2007.

[17] H. Torii and H. Nakagawa, "Infliximab monotherapy in Japanese patients with moderate-to-severe plaque psoriasis and psoriatic arthritis. A randomized, double-blind, placebocontrolled multicenter trial," Journal of Dermatological Science, vol. 59, no. 1, pp. 40-49, 2010.

[18] T.-F. Tsai, J.-C. Ho, M. Song et al., "Efficacy and safety of ustekinumab for the treatment of moderate-to-severe psoriasis: a phase III, randomized, placebo-controlled trial in Taiwanese and Korean patients (PEARL)," Journal of Dermatological Science, vol. 63, no. 3, pp. 154-163, 2011.

[19] J. Bagel, C. Lynde, S. Tyring, G. Kricorian, Y. Shi, and P. Klekotka, "Moderate to severe plaque psoriasis with scalp involvement: a randomized, double-blind, placebo-controlled study of etanercept," Journal of the American Academy of Dermatology, vol. 67, no. 1, pp. 86-92, 2012.

[20] A. Igarashi, T. Kato, M. Kato, M. Song, and H. Nakagawa, "Efficacy and safety of ustekinumab in Japanese patients with moderate-to-severe plaque-type psoriasis: long-term results from a phase $2 / 3$ clinical trial," Journal of Dermatology, vol. 39, no. 3, pp. 242-252, 2012.

[21] M. Zheng, X. J. Zhu, M. Song et al., "A randomized, doubleblind, placebo-controlled study of ustekinumab in Chinese patients with moderate to severe plaque psoriasis: LOTUS trial results," Journal of Dermatology, vol. 39, supplement 1, pp. 238239, 2012.

[22] S. R. Rapp, S. R. Feldman, M. L. Exum, A. B. Fleischer, and D. M. Reboussin, "Psoriasis causes as much disability as other major medical diseases," Journal of the American Academy of Dermatology, vol. 41, no. 3, pp. 401-407, 1999.

[23] C. Ferrándiz, A. García, A. J. Blasco, and P. Lázaro, "Costefficacy of adalimumab, etanercept, infliximab and ustekinumab for moderate-to-severe plaque psoriasis," Journal of the European Academy of Dermatology and Venereology, vol. 26, no. 6, pp. 768-777, 2012.

[24] R.-A. Greiner and L. R. Braathen, "Cost-effectiveness of biologics for moderate-to-severe psoriasis from the perspective of the Swiss healthcare system," European Journal of Dermatology, vol. 19, no. 5, pp. 494-499, 2009. 
[25] A. H. Anis, N. Bansback, S. Sizto, S. R. Gupta, M. K. Willian, and S. R. Feldman, "Economic evaluation of biologic therapies for the treatment of moderate to severe psoriasis in the United States," Journal of Dermatological Treatment, vol. 22, no. 2, pp. 65-74, 2011.

[26] S. Sizto, N. Bansback, S. R. Feldman, M. K. Willian, and A. H. Anis, "Economic evaluation of systemic therapies for moderate to severe psoriasis," British Journal of Dermatology, vol. 160, no. 6, pp. 1264-1272, 2009.

[27] G. L. Colombo, S. Di Matteo, K. Peris et al., "A cost-utility analysis of etanercept for the treatment of moderate-to-severe psoriasis in Italy," ClinicoEconomics and Outcomes Research, vol. 1, no. 1, pp. 53-59, 2009.

[28] R. J. B. Driessen, L. A. Bisschops, E. M. M. Adang et al., "The economic impact of high-need psoriasis in daily clinical practice before and after the introduction of biologics," British Journal of Dermatology, vol. 162, no. 6, pp. 1324-1329, 2010.

[29] C. C. Chi, "Evidence-based dermatology," Dermatologica Sinica, vol. 31, no. 1, pp. 2-6, 2013.

[30] Z. Ash, C. Gaujoux-Viala, L. Gossec et al., "A systematic literature review of drug therapies for the treatment of psoriatic arthritis: current evidence and meta-analysis informing the EULAR recommendations for the management of psoriatic arthritis," Annals of the Rheumatic Diseases, vol. 71, no. 3, pp. 319-326, 2012.

[31] A. Gottlieb, A. Menter, A. Mendelsohn et al., "Ustekinumab, a human interleukin 12/23 monoclonal antibody, for psoriatic arthritis: randomised, double-blind, placebo-controlled, crossover trial," The Lancet, vol. 376, no. 9752, p. 1542, 2010. 


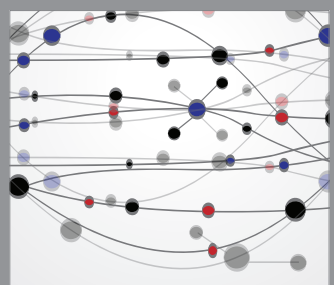

The Scientific World Journal
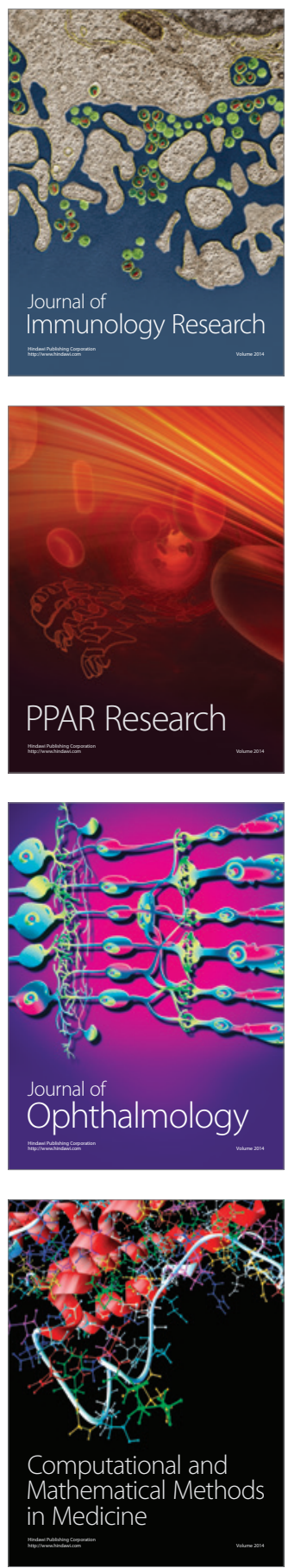

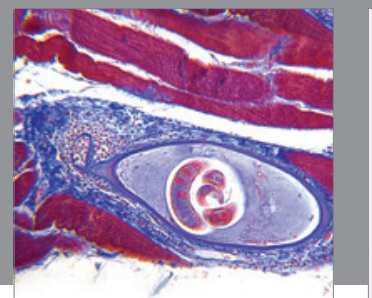

Gastroenterology

Research and Practice
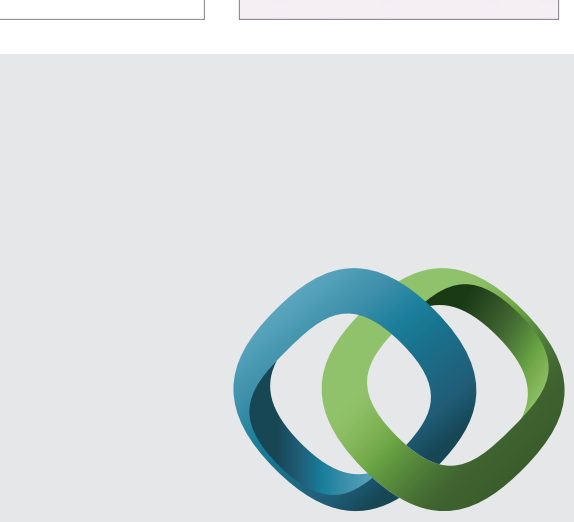

\section{Hindawi}

Submit your manuscripts at

http://www.hindawi.com
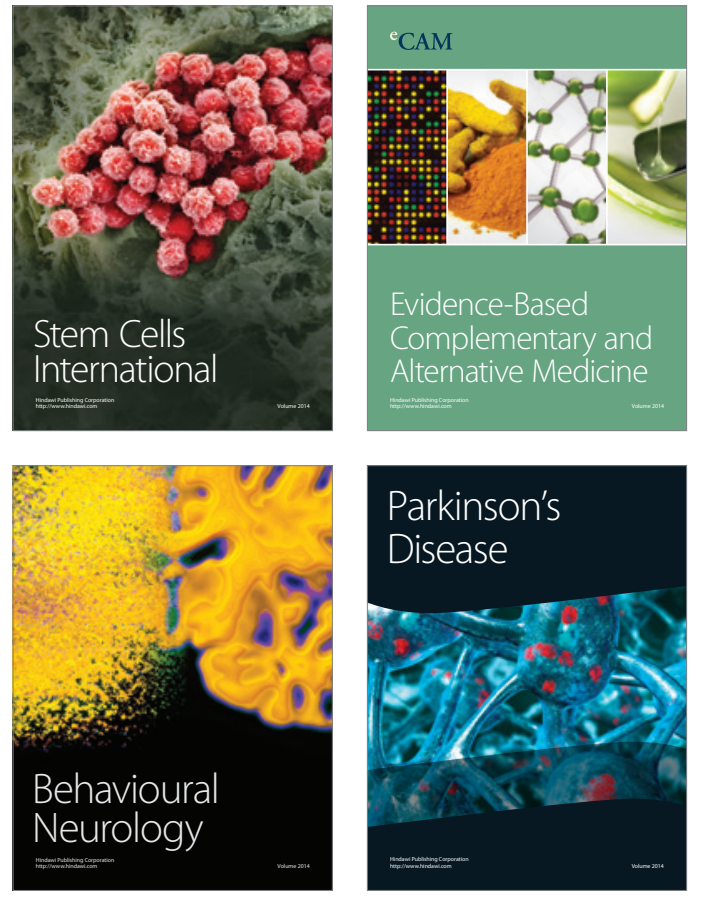
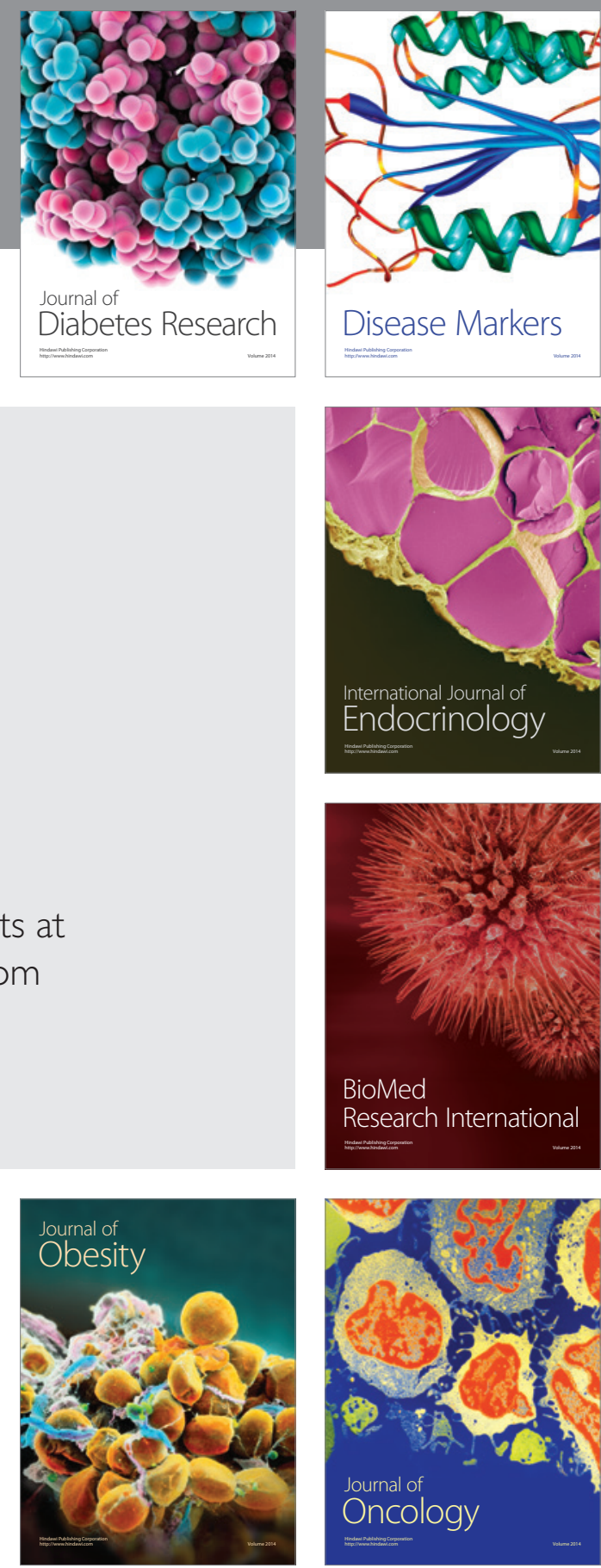

Disease Markers
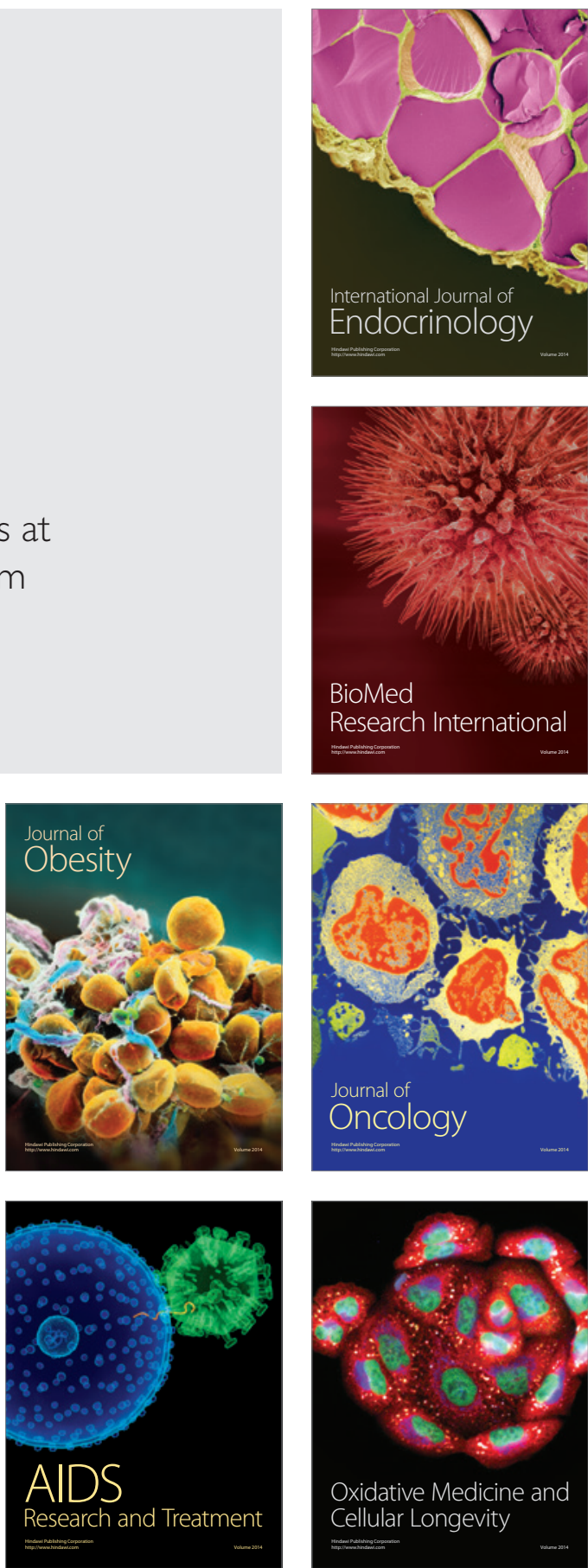